\title{
Renormalization of the One-Space Dimensional Yukawa Model by Unitary Transformation*
}

\author{
Paul Federbush \\ Department of Mathematics, University of Michigan, Ann Arbor, Michigan, 48104 \\ AND \\ BASILIS GIDAS
}

Department of Physics, Unirersity of Michigan, Ann Arbor, Michigan 48104

Received May 4, 1970

\begin{abstract}
A unitary transformation is used to renormalize the one-space dimensional Yukawa Model in a box. A momentum cut-off dependent sequence of unitary transformations is constructed such that weak limit matrix elements of the transformed Hamiltonian exist between a dense set of states as matrix elements of a symmetric operator.
\end{abstract}

In recent years a great deal of important progress has been made in the rigorous study of model relativistic quantum field theories [1]. Thus far the work has been mainly limited to superrenormalizable couplings. In such models requiring renormalization, Glimm has invented procedures employing nonunitary transformations of the Hamiltonian to carry out the renormalization [2]. On the other hand, two exactly solvable models that are renormalizable but not superrenormalizable, the derivative coupling model [3] and the massive Thirring model [4], are solved by unitary transformation [5]. In this paper, we set down a procedure for renormalizing the one-space dimensional Yukawa model by a unitary transformation. It is possible that some more difficult models can also be treated by similar methods.

We consider the one-space dimensional Yukawa model in a spatial box (interval) of length one with periodic boundary conditions and a momentum cut-off $\lambda_{2}$ :

$$
H_{\lambda_{2}}=H_{0 F}+H_{0 B}+V_{\lambda_{2}}+M_{\lambda_{2}}+E_{\lambda_{2}},
$$

* This work was supported in part by NSF grant GP 17523. 
where

$$
\begin{aligned}
& H_{0 F}=\sum_{y} \omega_{p}\left(a_{p}^{*} a_{p}+b_{p}^{*} b_{p}\right) \\
& H_{0 B}=\sum_{i} \mu_{k} d_{k}^{*} d_{k} \text {, } \\
& V_{\lambda_{2}}=g \int_{0}^{1} d x: \bar{\psi}_{\lambda_{2}}(x) \psi_{\lambda_{2}}: \varphi_{\lambda_{2}}(x) \\
& =\frac{g}{\sqrt{2}} \sum_{\substack{p_{1}, p_{2}, k \\
\left|p_{1}\right|,\left|p_{2}\right|,|k| \leqslant \lambda_{2}}} \frac{\delta_{p_{1}+p_{2}+k, 0}}{\sqrt{\mu_{k}}}\left(d_{k}^{*}+d_{-k}\right) \\
& \times\left\{\frac{\bar{u}\left(p_{1}\right) v\left(p_{2}\right)}{\sqrt{\omega_{1} \omega_{2}}} a_{p_{1}}^{*} b_{p_{2}}^{*}+\frac{\bar{u}\left(p_{1}\right) \underline{u\left(-p_{2}\right)}}{\sqrt{\omega_{1} \omega_{2}}} a_{p_{1}}^{*} a_{-p_{2}}\right. \\
& \left.-\frac{\bar{v}\left(-p_{1}\right) v\left(p_{2}\right)}{\sqrt{\omega_{1} \omega_{2}}} b_{p_{2}}^{*} b_{-p_{1}}+\frac{\bar{l}\left(-p_{1}\right) u\left(-p_{2}\right)}{\sqrt{\omega_{1} \omega_{2}}} b_{-p_{1}} a_{-p_{2}}\right\} \\
& M_{\lambda_{2}}=\frac{1}{2} \delta \mu_{\lambda_{2}}^{2} \int_{0}^{1} d x: \varphi_{\lambda_{2}}(x)^{2} \\
& \frac{1}{2} \delta \mu_{\lambda_{2}}^{2}=4 g^{2}\left(\ln \left(\frac{2 \lambda_{2}}{m}\right)-1\right) \\
& E_{\lambda_{2}}=\left(\frac{g}{\sqrt{2}}\right)^{2} \sum_{\substack{p_{1}, p_{2} \\
\left|p_{i}\right| \leqslant \lambda_{2}}} \frac{1}{\mu\left(p_{1}+p_{2}\right)\left(\omega_{1}+\omega_{2}+\mu\left(p_{1}+p_{2}\right)\right)}\left|\frac{\bar{u}\left(p_{1}\right) v\left(p_{2}\right)}{\sqrt{\omega_{1} \omega_{2}}}\right|^{2} .
\end{aligned}
$$

We follow the notation of [5]:

$$
\begin{gathered}
\omega_{p}=\sqrt{m^{2}+p^{2}}, \quad \mu_{k}=\sqrt{\mu_{0}^{2}+k^{2}} \\
\varphi_{\lambda_{2}}(x)=\frac{1}{\sqrt{2}} \sum_{\substack{k \\
|k| \leqslant \lambda_{2}}} \overline{\sqrt{\mu_{k}}}\left(d_{k} e^{i l i x}+d_{k}{ }^{*} e^{-i k x}\right) \\
\psi_{\lambda_{2}}(x)=\sum_{\substack{p \\
|p| \leqslant \lambda_{2}}} \frac{1}{\sqrt{\omega_{p}}}\left(a_{p} u(p) e^{i p x}+b_{p}{ }^{*} v(p) e^{i p x}\right) \\
\left|\frac{\bar{u}(\mathbf{p}) l\left(\mathbf{p}^{1}\right)}{\sqrt{\omega \omega^{I}}}\right|^{2}=\frac{\omega \omega^{1}-\mathbf{p p}^{1}-m^{2}}{\omega \omega^{1}} .
\end{gathered}
$$


We define operators $A_{k}\left(\lambda_{1}, \lambda_{2}\right)$ for $k \geqslant 0$ a possible boson momentum

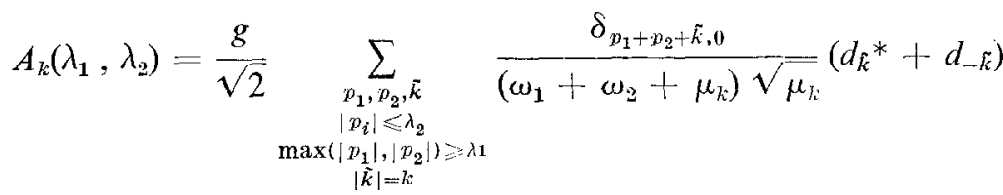

$$
\begin{aligned}
& \times\left\{\frac{\bar{u}\left(p_{1}\right) v\left(p_{2}\right)}{\sqrt{\omega_{1} \omega_{2}}} a_{p_{1}}^{*} b_{p_{2}}^{*}-\frac{\bar{v}\left(-p_{1}\right) u\left(-p_{2}\right)}{\sqrt{\omega_{1} \omega_{2}}} b_{-p_{1}} a_{-p_{3}}\right. \\
& +\epsilon\left(\left|p_{1}\right|,\left|p_{2}\right|\right) \frac{\bar{u}\left(p_{1}\right) u\left(-p_{2}\right)}{\sqrt{\omega_{1} \omega_{2}}} a_{p_{1}}^{*} a_{-p_{2}} \\
& \left.+\epsilon\left(\left|p_{1}\right|,\left|p_{2}\right|\right) \frac{\bar{v}\left(-p_{1}\right) v\left(p_{2}\right)}{\sqrt{\omega_{1} \omega_{2}}} b_{p_{2}}^{*} b_{-p_{1}}\right\},
\end{aligned}
$$

where

$$
\epsilon\left(\left|p_{1}\right|,\left|p_{2}\right|\right)=\left\{\begin{array}{rll}
0 & \text { if } & \omega_{1}=\omega_{2} \\
1 & \text { if } & \omega_{1}>\omega_{2} \\
-1 & \text { if } & \omega_{1}<\omega_{2}
\end{array}\right.
$$

We now define a unitary operator

$$
U\left(\lambda_{1}, \lambda_{2}\right)=e^{-. A_{k_{1}}\left(\lambda_{1}, \lambda_{2}\right)} e^{-A_{k_{2}}\left(\lambda_{1}, \lambda_{2}\right)} \cdots e^{-. A_{0}\left(\lambda_{1}, \lambda_{2}\right)},
$$

where $k_{1}, k_{2}, \ldots, 0$ is the decreasing sequence of possible nonnegative boson momentum values varying from $k_{1}=\lambda_{2}$ to zero.

We consider the domain $\mathscr{T}$ of states with finite number of particles with momentum spectra in a bounded range. We now claim there is a value $\lambda_{0}$ such that if $\lambda_{1} \geqslant \lambda_{\mathbf{0}}$, then for $\Phi, \Psi \in \mathscr{D}$ the limits

$$
\lim _{\lambda_{2} \rightarrow+\infty}\left(U\left(\lambda_{1}, \lambda_{2}\right) \Phi, H_{\lambda_{2}} U\left(\lambda_{1}, \lambda_{2}\right) \Psi\right)
$$

exist, converging to the matrix elements of a symmetric operator defined on $\mathscr{D}$

$$
\lim _{\lambda_{2} \rightarrow+\infty}\left(U\left(\lambda_{1}, \lambda_{2}\right) \Phi, H_{\lambda_{2}} U\left(\lambda_{1}, \lambda_{2}\right) \Psi\right)=\left(\Phi, \bar{H}\left(\lambda_{1}\right) \Psi\right)
$$

The verification is not difficult. We make three comments to explain the choice of $U\left(\lambda_{1}, \lambda_{2}\right)$ :

(1) The lower cut-off $\lambda_{1}$ is picked large to insure the convergence of the infinite series of terms generated in the expansion

$$
U\left(\lambda_{1}, \lambda_{2}\right)^{-1} H_{\lambda_{2}} U\left(\lambda_{1}, \lambda_{2}\right)
$$


As will be pointed out in (2), the number of types of terms of a given order $N$ only grows as $c^{N}$ for some $c$. If $\lambda_{1}$ is large enough, the $L_{2}$ norms of each term is estimated $\sim d^{N}$, where $d \rightarrow 0$ as $\lambda_{1} \rightarrow+\infty . \lambda_{1}$ must be picked large enough so that $\sum_{N} c^{N} d^{N}$ exists.

(2) The $A_{k}\left(\lambda_{1}, \lambda_{2}\right)$ have been constructed so that commutators of the $A$ 's contain no boson contractions. Thus in the expansion (17) the series is generated by commutators of fermion operators-fermion line contractions. The complexity of diagrams developable with no boson contractions is so limited that the types of diagrams to order $N$ can be estimated to be of order $c^{N}$.

(3) The arrangement of the $e^{-A_{k}}$ in order of decreasing momenta by a peculiar accident insures the convergence of otherwise divergent terms in the expansion (17). A simple such term is illustrated in Fig. 1.

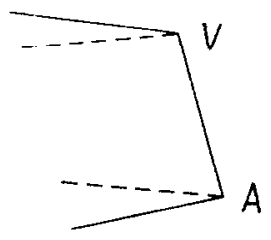

FigURE 1

As mentioned before, unitary transformations have been used to treat two nonsuperrenormalizable theories. There are certain obvious advantages (and disadvantages) to the use of unitary operators. It is possible that the procedure of Glimm can be combined with the use of a unitary transformation. The Yukawa model in two space dimensions is a good testing ground.

\section{REFERENCES}

1. J. Glimm AND A. JAfFe, Phys. Rev. 176 (1968), 1945. The $\left(\lambda_{\Phi}{ }^{4}\right)_{2}$ Quantum Field Theory Without Cut-offs II, III, to appear.

2. J. Glimm, Comm. Math. Phys. 5 (1967), 343; 10 (1968), 1.

3. B. SCHroer, Fortsch. Physik 11 (1963), 1.

4. P. Federbush, Phys. Rev. 121 (1961), 1247. Prog. Theor. Phys. 26 (1961), 148.

5. B. Gidas, Thesis, University of Michigan, Ann. Arbor, Mich., in preparation. 